\title{
Estimates of fission barrier heights for neutron-deficient Po to Ra nuclei produced in fusion reactions
}

\author{
Roman Sagaidak $^{\mathrm{a}}$ \\ Flerov Laboratory of Nuclear Reactions, Joint Institute for Nuclear Research, 141980 Dubna, Moscow region, Russia
}

\begin{abstract}
The cross section data for fission and evaporation residue production in fusion reactions leading to nuclei from Po to Ra have been considered in a systematic way in the framework of the conventional barrierpassing (fusion) model coupled with the statistical model. The cross section data obtained in very asymmetric projectile-target combinations can be described within these models rather well with the adjusted model parameters. In particular, one can scale and fix the macroscopic (liquid-drop) fission barrier heights (FBHs) for nuclei involved in the de-excitation of compound nuclei produced in the reactions. The macroscopic FBHs for nuclei from Po to Ra have been derived in the framework of such analysis and compared with the predictions of various theoretical models.
\end{abstract}

\section{Introduction}

The comparison of fission barrier heights (FBHs) calculated in a region between two neutron shells $(126 \leq$ $N \leq 184)$ reveals a large spread in their predictions for exotic neutron-rich nuclei [1]. At the same time, a reasonable description of FBHs close to the stability line (where model parameters are tuned to experimental data) is usually accomplished [2]. Large discrepancies between predictions of different models and FBHs derived for neutron-deficient nuclei produced in nuclear reactions were also observed earlier [3]. The differences between different calculations for extremely neutron-rich (hypothetical) Po nuclei reach $30 \mathrm{MeV}$. Such uncertainty results in completely different scenarios as far as the $r$-process termination by fission is considered [4]. So the study of the isotopic dependence of FBHs for nuclei far from the stability is one of the most important tasks in modern fission studies. Unfortunately, such studies are very di?cult for neutron-rich nuclei due to their very low production rates $[5,6]$. At the same time, the isotopic dependence of FBHs can be studied in the more readily accessible region of neutron-deficient nuclei produced in heavy ion (HI) fusion reactions.

\section{Statistical model considerations}

Within statistical model (SM) considerations production cross sections for the neutron-deficient heavy nuclei formed in very asymmetric projectile-target fusion reactions are mainly determined by level density parameters and $\mathrm{FBHs}$ for nuclei involved in compound nucleus $(\mathrm{CN})$ de-excitation chains. An analysis of the cross section data for evaporation residues (ERs) and fission with the use of the SM approximations [7] implies the FBH scaling of the liquid-drop (LD) barriers: $B_{\mathrm{f}}(L)=$ $k_{\mathrm{f}} B_{\mathrm{LD}}(L)-\Delta W_{\mathrm{gs}}$, where $k_{\mathrm{f}}$ is a scaling factor at the rotating LD barriers $B_{\mathrm{LD}}(L)$ [8] and $\Delta W_{\mathrm{gs}}$ is the ground

a e-mail: sagaidak@jinr.ru state shell correction. The macroscopic components of the barriers $B_{\mathrm{m}}=k_{\mathrm{f}} B_{\mathrm{LD}}$ derived from the analysis of Fr and Ra data $[9,10]$ are lower than any predictions $[2,3,11]$ (apart the values from [12]). The $B_{\mathrm{m}}$ values are about a smooth function of $N$, e.g., for Fr nuclei $k_{\mathrm{f}}$ is reduced from 0.85 to 0.8 , in going from $N=131$ to 117 (Fig. 1).

The data shown in Fig. 1 do not reveal any manifestation of the effect of the collective enhancement in the nuclear level density (CENLD). This effect is expected to be pronounced in the vicinity of the $N=126$ spherical shell as a decrease in the ER production cross sections, as it follows, e.g., from [5]. Such decrease is not observed in the ER cross section data obtained in the fusion experiments $[9,10]$ and, respectively, it is not expressed as a possible relative reduction in the $B_{\mathrm{m}}$ values (SM [7] does not take into account the CENLD effect in the calculated cross sections).

The similar analysis of the ER and fission data for Po nuclei in a wide region of $N(105 \leq N \leq 26)$ [14] shows a sizable reduction in the $B_{\mathrm{m}}$ values for neutron-deficient nuclei as compared to any predictions. This analysis includes ER data obtained in nearly symmetric and massive asymmetric projectile-target combinations leading to the most neutron-deficient nuclei. The possibility of quasi-fission (QF) effects [15] leading to the fusion probability $P_{\text {fus }}<1$ is not excluded in these reactions. Thus the comparison of the ER production cross sections obtained in the ${ }^{16} \mathrm{O}+{ }^{186} \mathrm{~W}$ and ${ }^{48} \mathrm{Ca}+{ }^{154} \mathrm{Sm}$ reactions leading to the ${ }^{202} \mathrm{~Pb}^{*} \mathrm{CN}[16,17]$ shows directly that $P_{\text {fus }}<$ 1 for the latter. These $P_{\text {fus }}$ values differ noticeably from those derived with the fission study [18] and obtained in the theoretical calculations [19] (Fig. 2).

\section{3. ${ }^{3,4} \mathrm{He}+\mathrm{Pb}, \mathrm{Bi}$ data analysis}

The ${ }^{3,4} \mathrm{He}+\mathrm{Pb}$, Bi reactions leading to $\mathrm{Po}$, At compound nuclei can be the most suitable for the $B_{\mathrm{m}}$ estimates in the light of the above mentioned QF effects observed in $\mathrm{HI}$ fusion reactions $(\mathrm{QF}$ may reduce the observable ER 


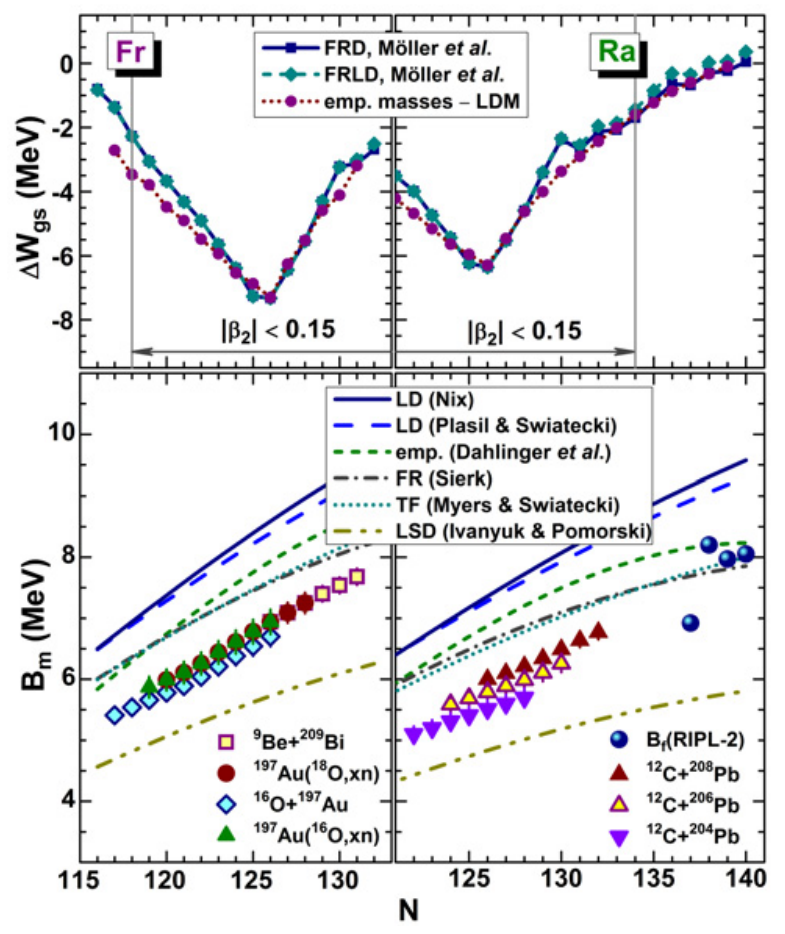

Figure 1. Bottom panels: the macroscopic fission barriers $B_{\mathrm{m}}$ for Fr and Ra nuclei derived with the SM [7] analysis of ER and fission cross section data obtained in the HI fusion reactions $[9,10]$ in comparison with the predictions $[2,3,11,12]$. Upper panels: the ground-state shell corrections $\Delta W_{\mathrm{gs}}$ in the vicinity of spherical nuclei corresponding to $\left|\beta_{2}\right|<0.15$ [13].

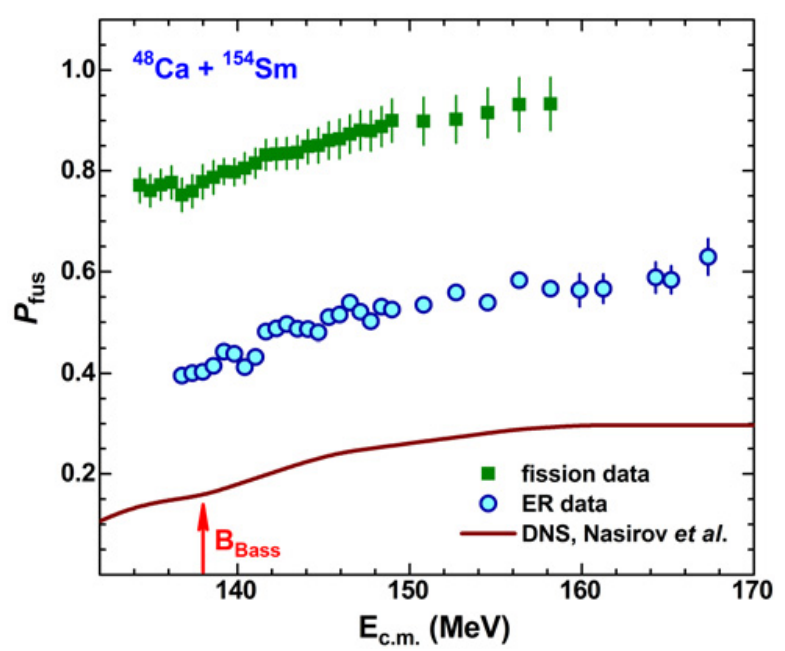

Figure 2. The fusion probabilities $P_{\text {fus }}$ extracted from the fission data obtained in the ${ }^{48} \mathrm{Ca}+{ }^{154} \mathrm{Sm}$ reaction [18] and similar values derived with the comparison of the ER cross sections measured in the same reaction and those obtained in ${ }^{16} \mathrm{O}+{ }^{186} \mathrm{~W}[16,17]$ (symbols). $P_{\text {fus }}$ calculated in the framework of the DNS concept [19] is also shown for comparison by solid line.

cross sections and the corresponding estimates of $B_{\mathrm{m}}$ [14]). The ${ }^{3,4} \mathrm{He}$ reactions have at least two advantages over the HI ones: a) much lower angular momenta are transferred to the compound nuclei formed in the light ion reactions, that ensures, along with relatively high excitation energies, more favorable conditions for the manifestation of the CENLD effect as compared to the HI reactions [14] characterized by very high $\mathrm{CN}$ rotation and $\mathrm{b}$ ) these

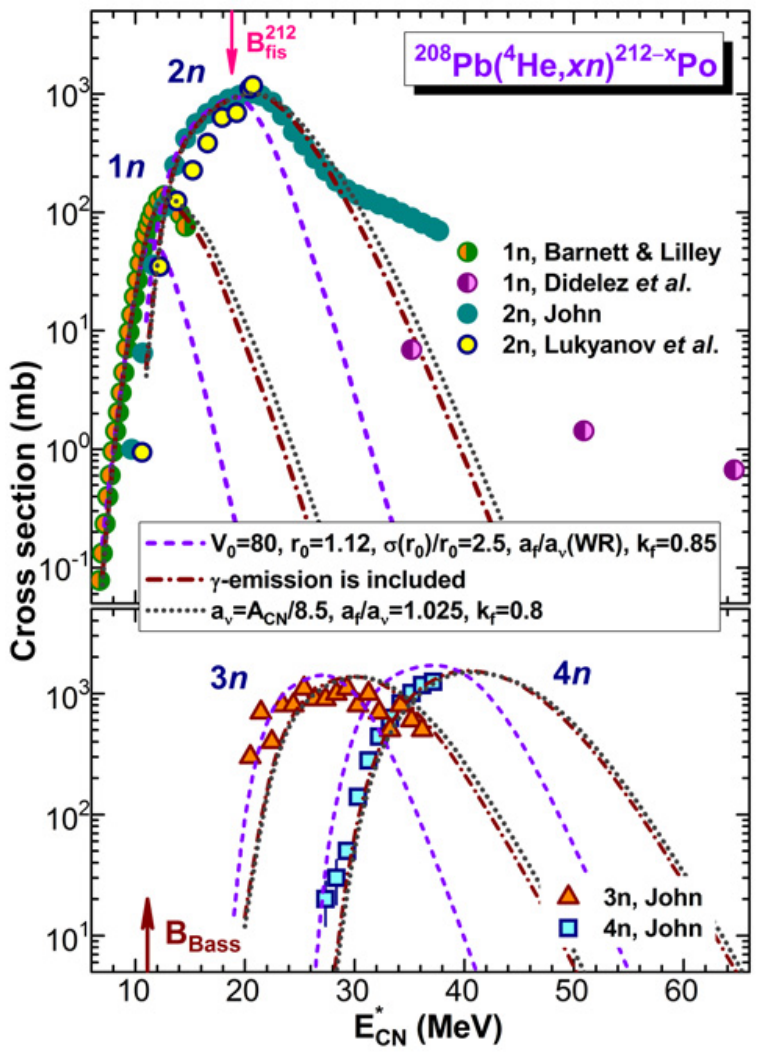

Figure 3. The production cross sections for ${ }^{211-208} \mathrm{Po}$ isotopes as measured by different authors in the ${ }^{208} \mathrm{~Pb}\left({ }^{4} \mathrm{He}, 1 n-4 n\right)$ reactions [21] (symbols). The evaporative excitation functions calculated with the SM [7] using the best choice of the nuclear potential parameters and different sets of the $\mathrm{CN}$-decay parameters as obtained in the present work (lines).

reactions do not reveal any indications of the QF effects, most likely due to the ultimate mass-asymmetry in the entrance channel of the reactions.

Earlier, FBHs of Po and At nuclei (along with other parameters determining the statistical decay of $\mathrm{CN}$ ) were derived using the fission data only [20]. At the same time, the available experimental data for the evaporative $\left({ }^{3,4} \mathrm{He}, x n\right)$ reactions [21] allow to perform the joint analysis of the ER and fission cross section data as was done for the HI data [14]. Such analysis implies the variation of the nuclear potential parameters which are sensitive to the ER cross sections in the vicinity of the fusion barrier, whereas fission data determine mainly the statistical parameters of the $\mathrm{CN}$ decay. Note that the fission data [20] were analyzed earlier with fusion cross sections calculated with the optical model.

The most representative ER data were obtained in the ${ }^{4} \mathrm{He}+{ }^{208} \mathrm{~Pb}$ reaction [21] as one can see in Fig. 3 for the $1 n-4 n$ evaporative channels. The $1 n$ and $2 n$ data allow choosing the main parameter values of the nuclear exponential potential [22] $\left(V_{0}, r_{0}\right.$ and $\sigma\left(r_{0}\right) / r_{0}$, the same as used in the previous HI data analysis [14]). The evaporating calculations indicate the importance of $\gamma$-emission at the $\mathrm{CN}$ de-excitation. The same calculations of the fission excitation functions (Fig. 4) are sensitive to the level-density and fission barrier scaling parameters $\left(a_{v}, a_{\mathrm{f}} / a_{v}\right.$ and $\left.k_{\mathrm{f}}\right)$ of SM [7]. At high energies the critical angular momentum for fusion $L_{\mathrm{cr}}$ becomes an important 


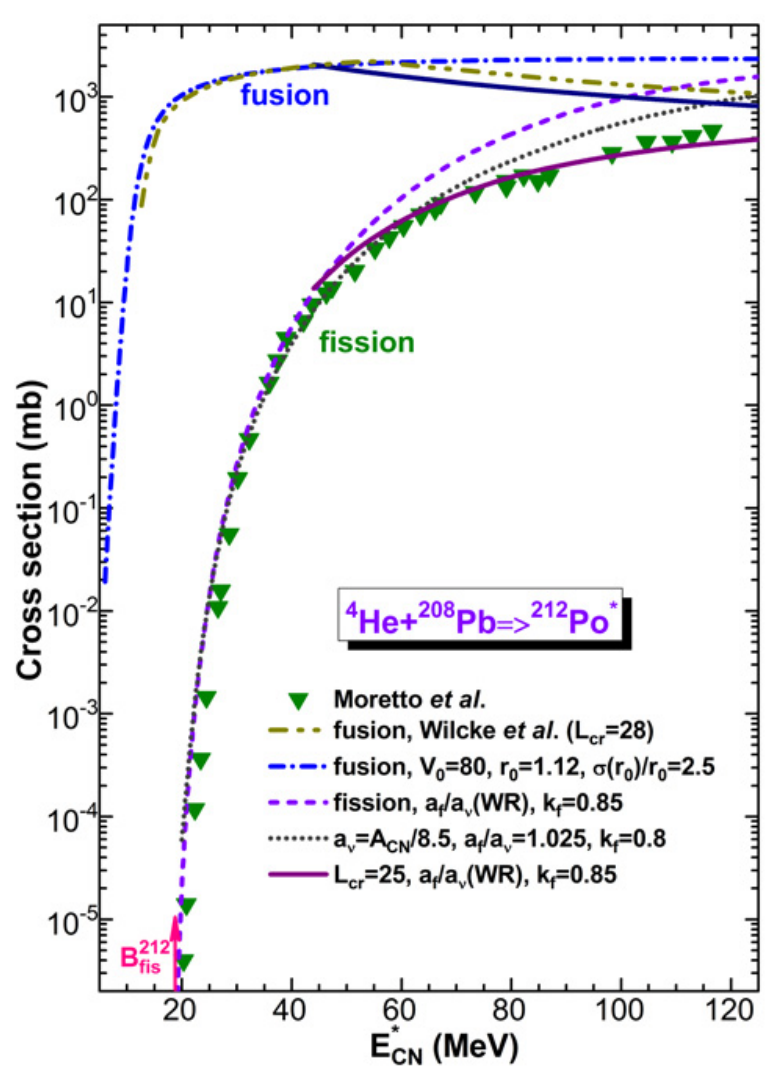

Figure 4. The fission cross section data obtained in the ${ }^{4} \mathrm{He}+{ }^{208} \mathrm{~Pb}$ reaction [20] (triangles) together with the fission excitation functions calculated in the same way as in Fig. 3 and with and without the use of $L_{\mathrm{cr}}$ (lines). Fusion cross sections calculated with the nuclear exponential potential [22] and those tabulated in [23] are also shown for the reference by specified lines.

Table 1. The parameter values of the nuclear exponential potential $\left(V_{0}, r_{0}\right.$ and $\left.\sigma\left(r_{0}\right) / r_{0}[22]\right)$ and the values of the critical angular momentum $L_{\mathrm{cr}}$ providing the best fit of the excitation functions calculated with $\mathrm{SM}$ to the ${ }^{3,4} \mathrm{He}+\mathrm{Pb}$, Bi data $[20,21]$.

\begin{tabular}{|l|c|c|c|c|c|}
\hline Projectile & Target & $\begin{array}{c}\boldsymbol{V}_{\mathbf{0}} \\
(\mathbf{M e V} / \mathbf{f m})\end{array}$ & $\begin{array}{c}\boldsymbol{r}_{\mathbf{0}} \\
(\mathbf{f m})\end{array}$ & $\begin{array}{c}\sigma\left(\boldsymbol{r}_{\mathbf{0}}\right) / \boldsymbol{r}_{\mathbf{0}} \\
(\boldsymbol{\%})\end{array}$ & $\begin{array}{c}\boldsymbol{L}_{\mathbf{c r}} \\
(\hbar)\end{array}$ \\
\hline${ }^{4} \mathrm{He}$ & $\begin{array}{c}208-206,204 \\
{ }^{209} \mathrm{Bb}\end{array}$ & 80 & 1.12 & 2.53 .5 & 2526 \\
\hline${ }^{3} \mathrm{He}$ & $\begin{array}{c}{ }^{208,207} \mathrm{~Pb} \\
{ }^{209} \mathrm{Bi}\end{array}$ & 60 & 1.25 & 8.5 & 16 \\
\hline
\end{tabular}

value determining the cross sections calculated for fission and for the $6 n-10 n$ evaporative channels.

In Table 1 the main parameter values of the nuclear exponential potential [22] and the values of the critical angular momentum $L_{\mathrm{cr}}$ providing the best fit of the excitation functions calculated with SM [7] to the data obtained in the ${ }^{3,4} \mathrm{He}+\mathrm{Pb}, \mathrm{Bi}$ reactions $[20,21]$ are shown.

In Table 2 the SM parameter values used for the description of the decay of Po and At compound nuclei are presented. These values, along with the data in Table 1, provide the best fit of the calculated excitation functions to the data $[20,21]$. The SM description of the $\mathrm{CN}$ decay with the level density parameters calculated according to [7] (designated as $a_{\mathrm{f}} / a_{v}(\mathrm{WR})$ in Figs. 3 and 4) seems to be preferable. The same approach was used in the previous analysis [14], although the excitation functions
Table 2. The SM parameter values $\left(a_{\mathrm{f}} / a_{v}\right.$ and $\left.k_{\mathrm{f}}\right)$ used for the description of the decay of Po and At compound nuclei. They give the best fit of the excitation functions calculated with SM [7] to the ${ }^{3,4} \mathrm{He}+\mathrm{Pb}, \mathrm{Bi}$ data [20,21]. The $L_{\mathrm{cr}}$ values [23] are also shown for the comparison with those presented in Table 1.

\begin{tabular}{|l|l|l|l|l|}
\hline Projectile & Target & $\boldsymbol{a}_{\mathbf{f}} / \boldsymbol{a}_{v}(\mathbf{W R})[7]$ & $\boldsymbol{k}_{\mathbf{f}}$ & $\boldsymbol{L}_{\mathbf{c r}}(\hbar)[\mathbf{2 3}]$ \\
\hline${ }^{4} \mathrm{He}$ & ${ }^{208-206,204} \mathrm{~Pb}$ & $1.0683-1.0655$ & \multirow{2}{*}{$\mathrm{He}$} & 28 \\
& ${ }^{209} \mathrm{Bi}$ & 1.0621 & & \\
\cline { 1 - 3 } & ${ }^{208,207} \mathrm{~Pb}$ & $1.0677,1.0670$ & & 18 \\
& ${ }^{209} \mathrm{Bi}$ & 1.0615 & & \\
\hline
\end{tabular}

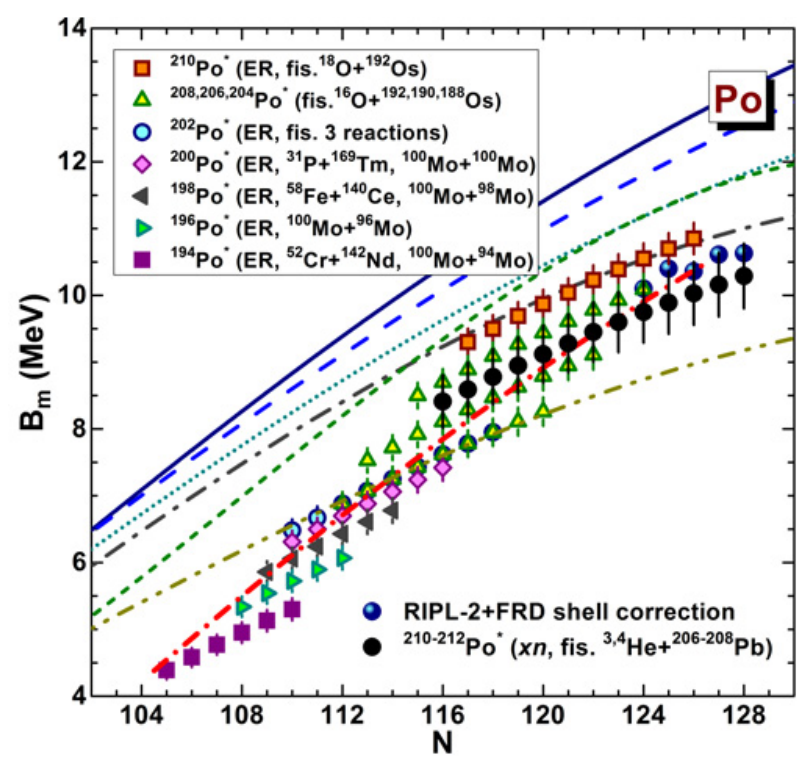

Figure 5. The macroscopic fission barriers $B_{\mathrm{m}}$ for Po nuclei derived with the SM [7] analysis of the ER and fission cross section data obtained in the HI fusion as previously described in [14] (different color symbols). The same is also but for the result of the present analysis of the cross section data obtained in the ${ }^{3,4} \mathrm{He}+\mathrm{Pb}$ fusion reactions $[20,21]$ (black circles). The predictions $[2,3,11,12]$ are shown for the comparison (the same lines and symbols as designated in Fig. 1).

calculated with $a_{v}=8.5 / A$ and $a_{\mathrm{f}} / a_{v}=1.05$ demonstrate very similar results.

In Fig. 5 the macroscopic fission barriers $B_{\mathrm{m}}$ derived for Po nuclei with the present analysis of the ER and fission cross section data obtained in the ${ }^{3,4} \mathrm{He}+\mathrm{Pb}$ fusion reactions $[20,21]$ are shown. The data are compared with the results of the previous analysis of HI fusion reaction data [14] and predictions $[2,3,11,12]$. As in the previous case [14], the macroscopic fission barriers derived in the region of $114<N \leq 128$ are lower than any predictions $[2,3,11]$ (except the values obtained in the framework of the LSD-model calculations [12]).

In Fig. 6 the macroscopic fission barriers $B_{\mathrm{m}}$ derived for At nuclei with the present analysis of the cross section data obtained in the ${ }^{3,4} \mathrm{He}+\mathrm{Bi}$ fusion reactions [20,21] are shown and compared with the predictions $[2,3,11,12]$. The barriers are lower than any predictions (apart the values from [12]), as obtained in the case of the results of the data analysis for Fr and Ra (Fig. 1).

Note that available ER cross section data obtained in the ${ }^{197} \mathrm{Au}\left({ }^{12} \mathrm{C}, x n\right){ }^{209-x} \mathrm{At}$ reactions (see [24] and Refs. therein) reveal a difference in the values corresponding the factor of 5-6. Such spread of the data leads to 


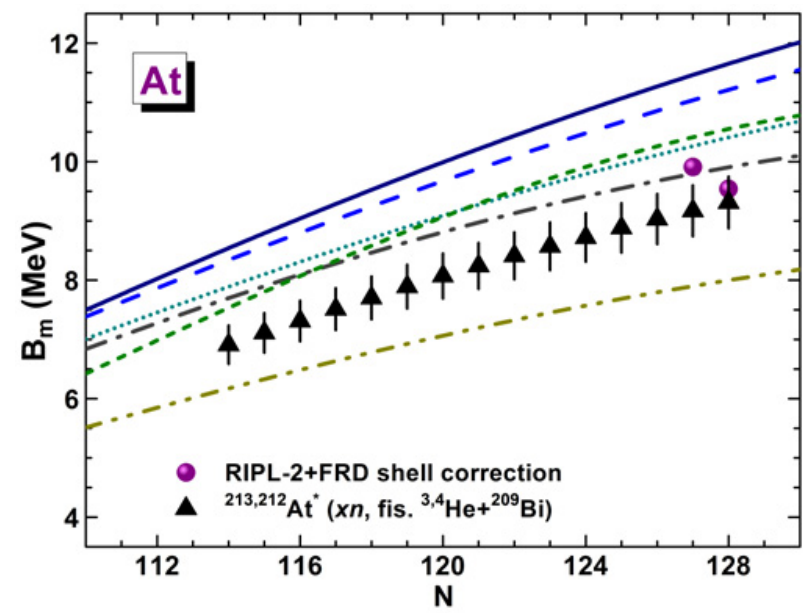

Figure 6. The same as in Fig. 5 but for the macroscopic fission barriers $B_{\mathrm{m}}$ of At nuclei, as derived with the analysis of the ER and fission cross section data obtained in the ${ }^{3,4} \mathrm{He}+\mathrm{Bi}$ fusion reactions $[20,21]$ (black triangles).

the spread in the 'adjusted $k_{\mathrm{f}}$ values' within the range of 0.5-1.0, according to the present SM [7] analysis.

\section{Summary}

The ER and fission cross sections data for the ${ }^{3,4} \mathrm{He}$ induced reactions leading to $\mathrm{Po}$ and At compound nuclei $[20,21]$ were analysed in the framework of the statistical model (SM) approximations [7]. With the corresponding choice of parameters, SM reproduces the cross section data for evaporation of neutrons and fission rather well. The macroscopic fission barriers derived for Po and At nuclei were found to be lower than those predicted with the models $[2,3,11]$. The exception is the barrier values predicted by the LSD model [12], which are distinctly lower than the derived ones. The same behaviour was earlier observed for Po barriers in the region of $114 \leq$ $N \leq 128$, as the result of the analysis of the HI fusion reaction data [14]. The barriers derived with the present study are in satisfactory agreement with those resulted in the previous analysis [14]. These findings indicate that ${ }^{3,4} \mathrm{He}$ data can be used to verify the results of the HI fusion data analysis applying a similar approach. The derived macroscopic fission barriers (along with other SM parameters used to describe the decay of compound nuclei) can be also used for the estimates of the fusion probability in the HI reactions induced by massive projectiles, which lead to Po and At compound nuclei.

\section{References}

[1] A. Mamdouh, J.M. Pearson, M. Rayet, F. Tondeur Nucl. Phys. A 679, 337 (2001)

[2] T. Belgya, O. Bersillon, R. Capote Noy et al., Handbook for calculations of nuclear reaction data, RIPL-2 (IAEA-TECDOC-1506, 2006), https://www-nds.iaea.org/ripl-2/
[3] M. Dahlinger, D. Vermeulen, K.-H. Schmidt, Nucl. Phys. A 376, 94 (1982)

[4] I.V. Panov, E. Kolbea, B. Pfeiffer et al., Nucl. Phys. A 747, 633 (2005)

[5] A.R. Junghans, M. de Jong, H.-G. Clerc et al., Nucl. Phys. A 629, 635 (1998)

[6] V.I. Zagrebaev, W. Greiner, Phys. Rev. C 87, 034608 (2013)

[7] W. Reisdorf, Z. Phys. A 300, 227 (1981); W. Reisdorf, M. Schädel, Z. Phys. A 343, 47 (1992)

[8] S. Cohen, F. Plasil, W.J. Swiatecki, Ann. of Phys. 82, 557 (1974)

[9] K.-T. Brinkmann, A.L. Caraley, B.J. Fineman et al., Phys. Rev. C 50, 309 (1994); L. Corradi, B.R. Behera, E. Fioretto et al., Phys. Rev. C 71, 014609 (2005); D.J. Hinde, M. Gupta, Phys. Rev. C 81, 064611 (2010)

[10] S. Santra, P. Singh, S. Kailas et al., Phys. Rev. C 64, 024602 (2001); D.J. Hinde, A.C. Berriman, R.D. Butt et al., J. Nucl. Rad. Sci. 3, 31 (2002); R.N. Sagaidak, G.N. Kniajeva, I.M. Itkis et al., Phys. Rev. C 68, 014603 (2003)

[11] J.R. Nix, Nucl. Phys. A 130, 241 (1969); F. Plasil, W.J. Swiatecki, in R. Vandenbosch, J.R. Huizenga, Nuclear fission (Academic Press, NY, 1973); A.J. Sierk, Phys. Rev. C 33, 2039 (1986); W.D. Myers, W.J. Swiatecki, Phys. Rev. C 60, 014606 (1999)

[12] F.A. Ivanyuk, K. Pomorski, Phys. Rev. C 79, 054327 (2009)

[13] P. Möller, J.R. Nix, W.D. Myers, W.J. Swiatecki, At. Data Nucl. Data Tables 59, 185 (1995)

[14] R.N. Sagaidak, A.N. Andreyev, Phys. Rev. C 79, 054613 (2009)

[15] J. Töke, R. Bock, G.X. Dai et al., Nucl. Phys. A 440, 327 (1985); B.B. Back, Phys. Rev. C 31, 2104 (1985)

[16] A.M. Stefanini, M. Trotta, B.R. Behera et al., Eur. Phys. J. A 23, 473 (2005)

[17] R.N. Sagaidak, EPJ Web of Conferences 21, 06001 (2012)

[18] G.N. Knyazheva, E.M. Kozulin, R.N. Sagaidak et al., Phys. Rev. C 75, 064602 (2007)

[19] A.K. Nasirov, G. Giardina, G. Mandaglio et al., Phys. Rev. C 79, 024606 (2009)

[20] F.D. Becchetti, K.H. Hicks, C.A. Fields et al., Phys. Rev. C 28, 1217 (1983); A.V. Ignatyuk, G.N. Smirenkin, M.G. Itkis et al., Sov. J. Part. Nucl. 16, 307 (1985); L.G. Moretto, K.X. Jing, R. Gatti et al., Phys. Rev. Lett. 75, 4186 (1995); Th. Rubehn, K. X. Jing, L.G. Moretto et al., Phys. Rev. C 54, 3062 (1996)

[21] Experimental Nuclear Reaction Data (EXFOR) http://www.nndc.bnl.gov/exfor/exfor.htm

[22] W. Reisdorf, F.P. Heßberger, K.D. Hildenbrand et al., Nucl. Phys. A 438, 212 (1985)

[23] W.W. Wilcke, J.R. Birkelund, H.J. Wollersheim et al., At. Data Nucl. Data Tables 25, 391 (1980)

[24] M. Cavinato, E. Fabrici, E. Gadioli et al., Phys. Rev. C 52, 2577 (1995); R. Joosten, J. Powell, F.Q. Guo et al., Phys. Rev. Lett. 84, 5066 (2000) 